\title{
CD44 expression in normal adrenal tissue and adrenal tumours
}

\author{
I Barshack, I Goldberg, D Nass, D Olchovsky, J Kopolovic
}

\begin{abstract}
Background-CD44 is a cell surface glycoprotein found on many normal cells, mainly lymphoid and epithelial. Normal cells usually express standard CD44 (CD44-S), whereas malignant tumours may express CD44 variant isoforms (CD44-V). CD44 expression has been described for neural crest derivatives. Characterisation of differences in CD44 expression may help in the diagnosis and differentiation of distinct adrenal tumours.

Aims-To examine CD44 expression in different layers of cortical cortex, in adrenal medulla, and in adrenal tumours.

Methods-CD44-S and CD44-V6 expression were studied in 12 cases of adrenal cortical adenoma, 3 of adrenal cortical carcinoma, 10 of pheochromocytoma, and 4 normal adrenal glands.

Results-CD44-V6 staining showed cytoplasmic expression in normal adrenal cortex and in cortical adenomas and carcinomas. Pheochromocytomas also showed CD44-V6 expression but in 5 of the 10 cases it was sparse, focal, and sometimes perinuclear. Strong membranous staining for CD44-S was observed in normal adrenal medulla. Analysis of CD44-S expression revealed differences between cortical adrenal tumours and pheochromocytomas. Ten of 12 cortical adenomas and 2 of 3 cortical carcinoma cells showed weak to moderate cytoplasmic staining, but all cases of pheochromocytoma had strong membranous staining.
\end{abstract}

Conclusions-Membranous

CD44-S

Department of Pathology, and Department of Medicine $A$ and

Endocrine Institute, Sheba Medical Center, Tel-Hashomer, affiliated to the

Tel-Aviv University, Sackler School of

Medicine, Israel

I Barshack

I Goldberg

D Nass

D Olchovsky

J Kopolovic

Correspondence to: Dr Barshack, The

Department of Pathology,

Chaim Sheba Medical

Center, Tel-Hashomer

52621, Israel.

Accepted for publication 10 October 1997 ymphoid and epithelial cells. It is kn mediate interaction with endothelium. This protein has many different forms, which are generated by alternative mRNA splicing and by post-translation modification. ${ }^{1}$ Normal cells usually express standard CD44 (CD44-S), while variant forms (CD44-V) may be expressed by malignant tumours. The identification of CD44 isoforms was found to be related to metastatic behaviour in some malignant tumours including those of the colon, ${ }^{12}$ stomach, ${ }^{3}$ and breast. ${ }^{4} \mathrm{CD} 44-\mathrm{V} 6$ was found to be a good marker for prognosis in breast cancer, ${ }^{4}$ and is expressed in advanced stages of colorectal carcinoma. ${ }^{2}$

The adrenal gland is composed of two different endocrine organs: the cortex, which originates in the mesoderm; and the medulla, which derives from the neuroectoderm. Adrenal cortical adenomas are characteristically small neoplasms composed of cells that have the appearance of zona glomerulosa and zona fasciculata of adrenal cortex. ${ }^{5}$ Pheochromocytoma is defined as a paraganglioma of the adrenal medulla, which is composed of specialised neural crest cells. ${ }^{6}$

CD44 expression was detected in the epithelium of murine adrenal cortex ${ }^{7}$ as well as in neural crest derivatives and neuroblastomas. $^{8}$

This study aimed at examining CD44 expression in different layers of the cortical cortex, in adrenal medulla, and in adrenal tumours. Characterisation of differences in CD44 expression may help in the diagnosis and differentiation of distinct adrenal tumours.

\section{Materials and methods}

Twelve cases of adrenal cortical adenoma, 3 of adrenal cortical carcinoma, 10 of pheochromocytoma, and 4 normal adrenal glands were selected from the surgical pathology material in the department of pathology of the Sheba Medical Center. Clinical data was retrieved from the patients' files. Formalin fixed, paraffin wax embedded tissues were sectioned at $4 \mu \mathrm{m}$ and placed on poly-1-lysine coated slides.

Immunohistochemical stainings were performed with labelled avidin biotin, using monoclonal anti-CD44 and polyclonal antiCD44-V6. Staining was enhanced by microwave pretreatment. Staining presence, pattern (membranous, cytoplasmic), and intensity $(0-3)$ in different identified cell types were evaluated by three observers. Discrepancies among observers were resolved following discussion around a multiviewer microscope. All adenoma cases were searched for normal adrenal tissue, which was found in seven of 12 cases. Attention was paid to different cell types in the adrenal cortex and medulla.

\section{Results}

EXPRESSION OF CD44-S AND CD44-V6 VARIANT PROTEIN IN NORMAL ADRENAL TISSUE

The cell distribution of CD44-S and CD44-V6 was assessed in immunohistochemical studies. Changes were observed in CD44-V6 expression between different parts of the adrenal cortex. Cells of the zona glomerulosa had the most prominent cytoplasmic staining. The adrenal 


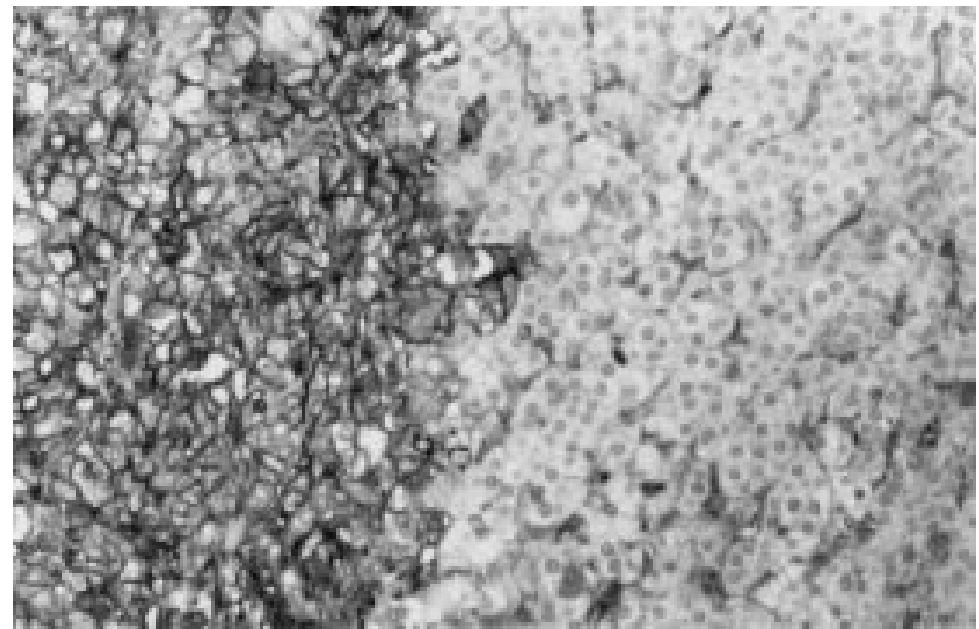

Figure 1 Membranous staining of CD44-S in the medulla (left) compared with the adrenal cortex (right).

Table 1 CD44-S membranous expression in cortical adenoma, carcinoma, and pheochromocytoma

\begin{tabular}{llll}
\hline Pathological diagnosis & $\begin{array}{l}\text { Number of } \\
\text { cases }\end{array}$ & CD44-S expression & $\begin{array}{l}\text { Hormone secreting (number of } \\
\text { cases) }\end{array}$ \\
\hline Adenoma & 10 & - & Cortisol (3) Aldosterone (3) \\
& 2 & +2 & Non (4) \\
Carcinoma & 2 & - & Cortisol (1) Non (1) \\
Pheochromocytoma & 1 & +2 & Cortisol (1) Non (1) \\
& 8 & +3 & \\
\hline
\end{tabular}

$\star$ Intensity of membranous staining: -, very weak/equivocal; +1 , weak; +2 , moderate; +3 , strong.

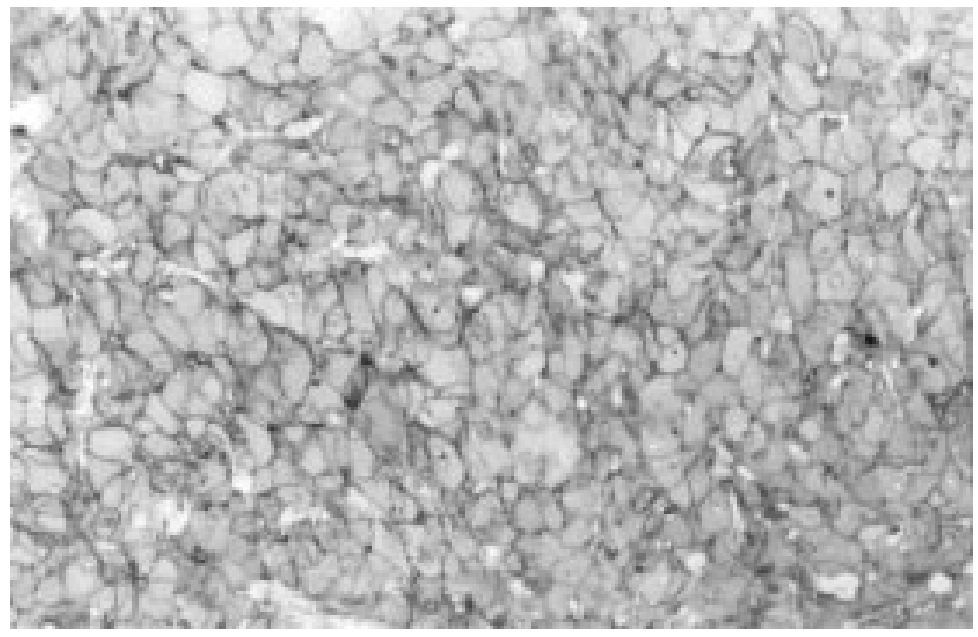

Figure 2 CD44-S membranous staining in pheochromocytoma cells.

cortex showed weak cytoplasmic expression of CD44-S. In contrast to this restricted expression, the adrenal medulla showed strong membranous staining of CD44-S (fig 1).

EXPRESSION OF CD44-S AND CD44-V6 VARIANT PROTEIN IN ADRENAL TUMOURS

Adrenal cortical adenomas had cytoplasmic expression of CD44-V6 and CD44-S. Two exceptions were found also to have CD44-S membranous staining (table 1). Adrenal cortical carcinoma cells also had cytoplasmic expression of CD44-V6 and CD44-S, whereas only one of the three cases also showed membranous staining of CD44-S. CD44-V6 expression was demonstrated in the pheochro- mocytoma cells; however, in five of the 10 cases it was sparse, focal, and mainly perinuclear.

Compared with the adrenal cortical adenomas and cortical carcinomas that were examined, all pheochromocytoma cells showed strong membranous expression of CD44-S (fig 2).

Of the 12 cortical adenoma patients, four were cortisol secretors, three aldosterone secretors, and the others non-secretors. The two exceptional cases with CD44-S membranous staining were not different from the rest of the group (table 1). The sole case of carcinoma with membranous staining for CD44-S was a cortisol and androgen secretor.

\section{Discussion}

CD44 is an integral membrane glycoprotein, functioning as a lymphocyte homing receptor. It has been found to be expressed in malignant tumours including colon, stomach, and breast carcinomas, as well as in many normal tissues. ${ }^{2-4}$ Combaret et al detected strong CD 44 expression in ganglioneuroblastomas, ganglioneuromas, and normal neuroblasts migrating in the fetal adrenal gland. ${ }^{8}$ They also observed CD44 expression in Schwann and ganglion cells. $^{8}$

Occasionally, pheochromocytomas may be difficult to distinguish from cortical adenomas, as pheochromocytoma and adrenal cortical adenoma may have similar histological appearances. ${ }^{9}$ Adrenal cortical adenoma cells usually take the form of zona glomerulosa, zona fasciculata or both. Immunohistochemically, they show expression of low molecular weight keratin, rarely of vimentin, and various steroid hormones and enzymes. ${ }^{10}$ Pheochromocytoma can be defined as a paraganglioma of the adrenal medulla and typically exhibits positivity for catecholamines, neuron specific enolase, chromogranins, synaptophysin, and opioid peptides. ${ }^{11}$ Pheochromocytomas, especially those with lipid degeneration, may mimic adrenal cortical adenomas grossly and microscopically. ${ }^{12}$ Similar intracytoplasmic hyaline globules can be detected in pheochromocytomas and in cortical neoplasms. ${ }^{14}{ }^{15}$ If the pheochromocytoma also happens to be an adrenocorticotrophin hormone secretor the distinction may become more difficult. ${ }^{9}$

In this study, cytoplasmic staining for CD44-V6 was detected in the adrenal cortex that differed in intensity between different zones of adrenal cortex. Adrenal cortical adenomas and carcinomas showed CD44-V6 and CD44-S cytoplasmic staining. Two exceptions of adrenal cortical adenoma and one of cortical carcinoma also showed CD44-S membranous staining. No clinical differences were observed between these cases and the rest of the group. Further investigation using larger numbers of cases of carcinoma is needed to establish the pattern of staining and to determine the correlation between CD44 expression and tumour progression in cortical carcinoma cells.

In this study, strong membranous CD44-S expression was detected on medulla cells of the normal adrenal. A strong membranous staining 
was also shown by all 10 cases of pheochromocytoma. Hence, cytoplasmic staining of CD44-S favours the diagnosis of cortical adenoma, whereas positive membranous staining is characteristic of pheochromocytoma. Therefore, pheochromocytoma, which probably originates in the neural crest, maintains its CD44 expression. Moreover, strong membranous CD44 staining of pheochromocytoma may help distinguish this tumour from adrenal cortical adenomas.

1 Tanabe KK, Ellis LM, Saya H. Expression of CD44R1 adhesion molecule in colon carcinomas and metastases. Lancet 1993;341:725-6.

2 Wielenga VJM, Heider $\mathrm{KH}$, Offerhaus GJA, et al. Expression of CD44 variant proteins in human colorectal cancer is related to tumor progression. Cancer Res 1993;53:47546.

3 Washington $\mathrm{K}$, Gottfried MR, Telen MJ. Expression of the cell adhesion molecule CD44 in gastric adenocarcinoma. Hum Pathol 1994;25:1043-9.

4 Kaufmann M, Heider KH, Sinn HP, et al. CD44 variant exon epitopes in primary breast cancer and length of survival. Lancet 1995;345:615-19.

5 Cote RJ, Cordon-Cardo C, Reuter VE, et al. Immunopathology of adrenal and renal cortical tumors. Coordinated change in antigen expression is associated with neoplastic conversions in the adrenal cortex. Am f Pathol 1990;136: $1077-84$.
6 Bravo El, Grifford RW. Pheochromocytoma. Diagnosis, Bravo El, Grifford RW. Pheochromocytoma. Diagnosis, localization

7 Kennel SJ, Lankford TK, Foote LJ, et al. CD44 expression on murine tissues. F Cell Sci 1993;104:373-82.

8 Combaret V, Coll JL, Favrot MC. Expression of integrin and CD44 adhesion molecules on neuroblastoma: the relation to tumor aggressiveness and embryonic neural-crest differentiation. Invasion Metastasis 1994-1995;14:156-63.

9 Lack EE. Tumors of the adrenal gland and extra-adrenal paraganglia. Washington, DC: American Forces Institute of Pathology, 1997 .

10 Sasano H, White PC, New MI, et al. Immunohistochemical localization of cytochrome P-450 in human adrenal cortex and its relations to endocrine function. Hum Pathol 1988;19:181-5.

11 Bostwick DG, Null WE, Holmes D, et al. Expression of opiod peptides in tumors. N Engl f Med 1987;317:143943.

12 Ramsey JA, Asa SL, van Nordstrand AW, et al. Lipid degeneration in pheochromocytomas mimicking adrenal cortical tumors. Am f Surg Pathol 1987;11:480-6.

13 Unger PD, Cohen JM, Thung SN, et al. Lipid degeneration in a pheochromocytoma mimicking an adrenal cortical in a pheochromocytoma mimicking an adre
tumor. Arch Pathol Lab Med 1990;114:892-4.

14 Linnolia RI, Keiser HR, Steinberg SM, et al. Histopathology of benign versus malignant sympathoadrenal paragangliomas: clinicopathologic study of 120 cases including unusual histologic features. Hum Pathol 1990;21: $1168-80$.

15 Lack EE, Mulvihill JJ, Travis WD, et al. Adrenal cortical neoplasms in the paediatric and adolescent age group. Clincopathologic study of 30 cases with emphasis on epidemiological and prognostic factors. Pathol Annu 1992; 27:1-53. 